LBNL-56559

CBP Note-637

\title{
Feasibility Analysis for Attosecond X-ray Pulses at FERMI@ELETTRA Free
} Electron Laser*

\author{
Alexander Zholents \\ Lawrence Berkeley National Laboratory \\ University of California, \\ Berkeley, California 94720
}

September 2004

* This work was supported by the Director, Office of Science of the U. S. Department of Energy under Contract No. DE-AC03-76SF00098. 


\begin{abstract}
We present preliminary analysis for the feasibility of the attosecond x-ray pulses at a proposed FERMI@ELETTRA free electron laser (FEL) [1]. In part 1 we restrict ourselves to minimal modifications to the proposed FEL and consider a scheme for attosecond x-ray production which can be qualified as a small add-on to a primary facility. We demonstrate that at 5-nm wavelength our scheme is capable for production of pulses with an approximate duration of 100 attoseconds at approximately $2 \mathrm{MW}$ peak power and with an absolute temporal synchronization to a pump laser pulse. In part 2 we propose to use an FEL amplifier seeded by a VUV signal and to follow it by the scheme for attosecond $\mathrm{x}$-ray production described in part 1.
\end{abstract}




\section{Part 1}

In this work we closely follow a proposal published in [2]. The method described there for obtaining of attosecond $\mathrm{x}$-ray pulses involves the use of ultra-relativistic electron beam, a few-cycle, intense optical laser pulse and an intense pulse of the coherent $\mathrm{x}$-ray radiation, together with a number of magnetic undulators and transport elements. The proposed FERMI@ELETTRA facility will have most of these components and, therefore, seems to be ideally suited for production of isolated, attosecond duration, short-wavelength x-ray pulses.

In our study we use the following electron beam parameters [1]: energy of $1 \mathrm{GeV}$, normalized emittance of $1.5 \mathrm{~mm}$-mrad, energy spread of $0.5 \mathrm{MeV}$, bunch length of 320 fs, and peak current of $1.25 \mathrm{k} \mathrm{A}$. Comparing to Ref. [1], we increase two times the bunch length and reduce two times the energy spread and the peak current. We increase the bunch length in order to have some "virgin" electrons (i.e. electrons untouched by any interaction with the light in the upstream part of the machine) for a production of an attosecond x-ray pulse.

Figure 1 schematically shows a process of generation of attosecond pulses proposed for the FERMI@ELETTRA facility. On the left is a harmonic cascade free electron laser (HC FEL) producing 50-100 fs, 50-MW peak power pulses of coherent radiation at $10-\mathrm{nm}$ wavelength. After exiting the HC FEL, an achromatic bend inserts the electrons into a two-period wiggler magnet called "800-nm modulator". Simultaneously, a 800-nm wavelength, 1-mJ, 5-fs laser pulse enters this wiggler and co-propagates with the electrons. The technical feasibility of such optical pulses has already been proven [3]. The relative timing between the arrival of the electron beam and the optical pulse is set such that the latter temporally overlaps a short section of "virgin" electrons.

We presume that the few-cycle laser pulse is originated from the same laser source as the seed laser pulse for HC FEL. This permits tight synchronization between the few-cycle laser pulse and the HC FEL's, 10-nm pulse. Since the "virgin" electrons belongs to the same electron bunch as those producing the HC FEL's 10-nm pulse, one can thus ensure temporal synchronization between each of these three pulses.

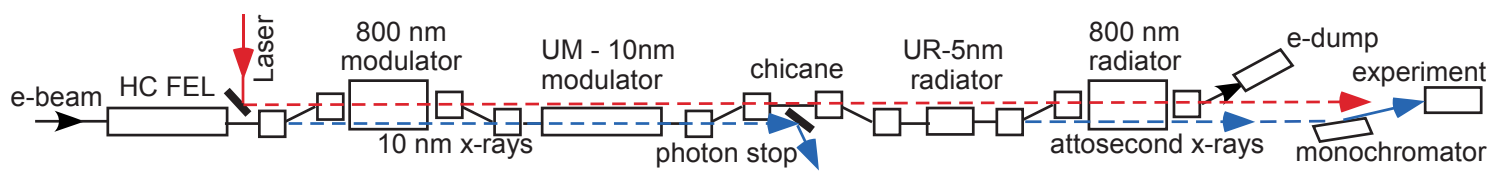

Figure 1. A schematic of the components involved in attosecond x-ray pulse production.

The carrier-envelope phase of the few-cycle laser pulse is adjusted so that the peak electric field appears at the peak of the envelope when the laser pulse passes the wiggler center. The wiggler's period of $10 \mathrm{~cm}$ and undulator parameter $K=11$ are adjusted such that fundamental FEL resonance occurs at the laser wavelength $800 \mathrm{~nm}$. The interaction with the laser light in the wiggler then produces a time-dependent electron energy modulation as shown in Figure 2. For the laser pulse parameters mentioned above, we expect central peak energy gain to be $\sim 15 \mathrm{MeV}$ and a factor of 1.35 times larger than those of its nearest neighbors. This relative difference is important when considering the $10-\mathrm{nm}$ energy modulation to be induced in a following undulator. 


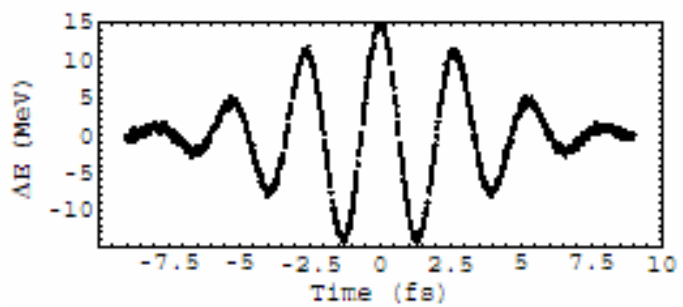

Figure 2. The calculated energy modulation of the electrons along the electron bunch produced in the interaction with a few-cycle, 800 -nm laser pulse in the wiggler magnet.

A second achromatic and isochronous bend after the wiggler magnet returns the electrons back to the original axis, while the 800 -nm laser pulse continues to propagate along a parallel, offset path. The electrons now enter a $1.8 \mathrm{~m}$ long undulator-modulator (UM) (50 periods, period length of $3.66 \mathrm{~cm} \mathrm{[1],} \mathrm{not} \mathrm{shown} \mathrm{to} \mathrm{scale} \mathrm{in} \mathrm{Figure} \mathrm{1)} \mathrm{which}$ serves as an energy modulator at $10-\mathrm{nm}$ wavelength. The coherent, $50-100 \mathrm{fs}$ long, $\sim 50$-MW peak power, 10-nm output pulse from the HC FEL co-propagates in the UM with electrons and arrives simultaneously with those electrons that experienced the strong energy modulation at $800-\mathrm{nm}$. The undulator parameter $K$ of the UM is tuned such that only those electrons very near the peak of the 800 -nm energy modulation have the correct energy for resonant FEL interaction with the 10-nm light. The other electrons fall outside the energy bandwidth of the UM and are not significantly modulated. We note that the UM is shorter than one gain length of self-amplified spontaneous emission (SASE) so there is little SASE action there (which otherwise would produce unwanted microbunching at 10-nm wavelength throughout the 320-fs long electron bunch).

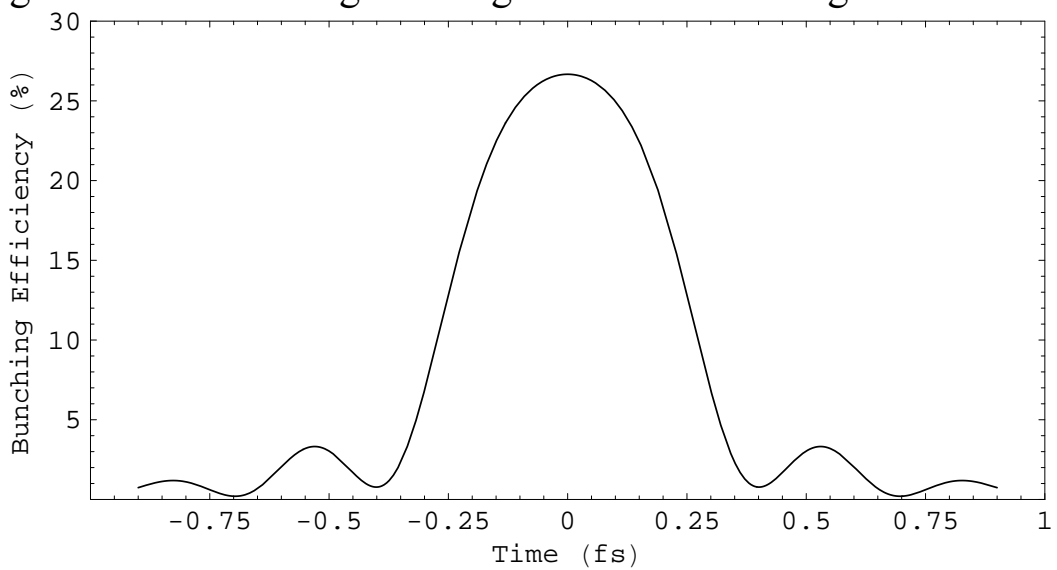

Figure 3. Bunching efficiency at 5-nm wavelength versus time along the electron bunch.

Downstream of the UM the electrons enter a chicane with a time-of-flight parameter $R_{56}=1000 \mathrm{~nm}$ which induces strong microbunching at 10-nm wavelength and at higher harmonics. We calculate bunching efficiency at a second harmonic, i.e 5-nm, using procedure described in [2] and plot it in Figure 3. In this calculation we assume 50 MW peak power for the output radiation of the HC FEL at 10-nm, and a detuning of the UM from the FEL resonance equivalent to the $4 \mathrm{MeV}$ energy off-set.

In the middle of the chicane, the electron beam orbit is separated transversely from the path of the x-ray light. This permits use of a photon stop to block/reflect all light 
coming to this point, which is important for obtaining maximum contrast of the attosecond $\mathrm{x}$-ray pulse over the background radiation.

After the chicane, the electrons proceed to an undulator-radiator (UR) tuned for coherent emission from the bunched electrons at wavelength 5-nm. Here we consider a linearly-polarized UR with period $2 \mathrm{~cm}$ and undulator parameter $K=1.4$ (not shown to scale in Figure 1), but note that a helically-polarized UR can also be used. The predicted radiation field intensity is shown in Figure 4. There is destructive interference (due to temporal variation of bunching phase) occurring between waves emitted by microbunches on opposite sides of the bunching peak. This effect explains shorter duration of the radiation pulse in Figure 4 comparing to the temporal bunching structure in Figure 3. For a given temporal bunching structure, the temporal structure of the pulse in Figure 4 depends upon the UR length and detuning from the FEL resonance. We found that the UR with 17 periods and detuning equivalent to $4 \mathrm{MeV}$ energy off-set gives the shortest pulse $\sim 120$ fs (FWHM).

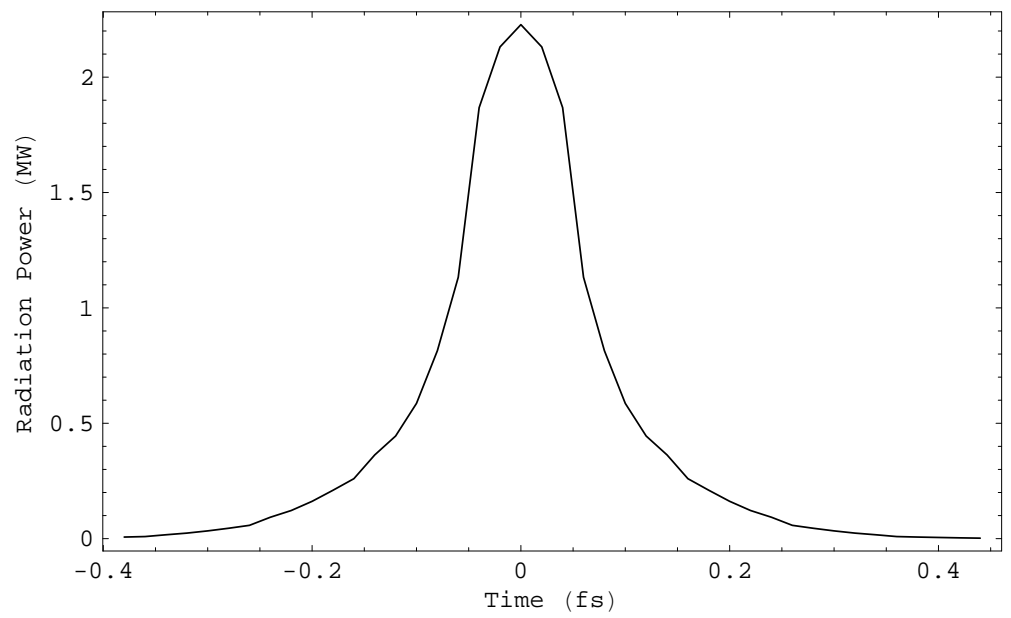

Figure 4. Predicted attosecond pulse power at 5-nm wavelength from a radiator with 17 periods.

For a given electron beam emittance and the x-ray wavelength of 5-nm, the source size of the attosecond radiation is defined by the electron beam size which is approximately $40 \mu \mathrm{m}$ (at one sigma level) for an assumed beta-function of $2 \mathrm{~m}$ in the UR. The attosecond radiation spreads out within the solid angle $\sim \pi 10^{-10} \mathrm{sr}$ which is more than two orders of magnitude smaller than the solid angle of the electron spontaneous emission in the UR at 5-nm. Thus the collimator can significantly improve the contrast of the attosecond pulse.

Figure 4 gives a conservative estimate for radiation intensity which can be improved using tighter electron beam focusing in the UR. However, this must be considered in the context of a design of the entire system. We note that the temporal structure of the radiation pulse is given rather accurately.

The last component in the Figure 1 is the " $800-n m$ radiator". Those electrons which underwent the 800 -nm energy modulation can be strongly bunched at this wavelength via an achromatic bend following the UR with a relatively large $R_{56}$ coefficient. They can then emit a few-cycle, sub-microjoule coherent pulse at $800-\mathrm{nm}$ in a few-period wiggler magnet shown as $800-\mathrm{nm}$ radiator before heading to the dump. This 
secondary pulse (which is automatically temporally synchronized with the 5-nm attosecond pulse) can then be cross-correlated with the original 800-nm modulating laser pulse to provide a feedback for a fine tuning of the synchronization between the pump and probe pulses.

\section{Part 2}

A technique of high harmonic generation (HHG) using a hollow-fiber and selfguided intense laser beam in a long gas cell has been actively pursuing in different laboratories (see, for example, [4-7 ]). Short, coherent VUV pulses produced with this technique had already been reached spectacular peak power levels of the order of 10-MW at 30-nm wavelength, i.e. $0.3-\mu \mathrm{J}$ pulse energy in a less than 35-fs long pulse as reported in [8]. Here we propose to use this pulse as a seed signal for an FEL amplifier and harmonic generation at 10 -nm wavelength which can be followed by the above described scheme for a generation of attosecond pulses. (The use of a short wavelength radiation produced by HHG to seed the FEL had been also considered in the MIT's X-ray laser project [9] and more recently in [10]).

Namely, we propose to co-propagate the HHG-made pulse with a $1 \mathrm{GeV}$ electron beam in the undulator and create a time-dependent energy modulation of electrons at 30nm wavelength. The analysis shows that a 30-fs long HHG's pulse with a 5-MW peak power will induce a $1.5 \mathrm{MeV}$ energy modulation in a short section of the electron beam while co-propagating 150 periods of the undulator with $K=3.2$ and period length of 3.66 $\mathrm{cm}$. Following energy modulation, the electrons are bunched in the downstream chicane giving the rise to spatial harmonics of the peak current in the energy modulated section of the electron beam. Assuming the electron beam uncorrelated energy spread of $0.5 \mathrm{MeV}$ and the normalized emittance of $1.5 \mathrm{~mm}-\mathrm{mrad}$, we calculate $\sim 10 \%$ bunching efficiency at a 3-rd harmonic of the modulation frequency (10-nm wavelength). This is sufficient for electrons with an original $1.25 \mathrm{kA}$ peak current (before bunching) to be able to radiate $\sim 50 \mathrm{MW}$ peak power from the undulator-radiator with period of $3.66 \mathrm{~cm}$ and 100 periods tuned for coherent emission from the bunched electrons at 10-nm. Thus, use of the HHGmade signal permits to substitute the HC FEL with just one cascade of the FEL amplifier and harmonic generation.

From this point we propose to follow the scheme for attosecond generation described in Part 1. We note that the few-cycle laser pulse used in attosecond generation and the intense laser pulse needed for HHG can be originated from the same laser source thus permitting a tight synchronization between the HHG's output pulse and those electrons that experienced the strong energy modulation at $800-\mathrm{nm}$.

Acknowledgement. We thank Fulvio Parmigiani for a proposed theme for our study.

\section{References}

[1] R.J. Bakker, et. al., in Proc. of European Particle Accelerator Conference 2004.

[2] A.A. Zholents, W.M. Fawley, Phys. Rev. Lett., 92, 224801 (2004).

[3] S. Sartania, Opt. Lett., 22, 1562(1997).

[4] A. Runquist, et. al., Sceince, 280, 1412(1998).

[5] E. Constant, et. al., Phys. Rev. Lett., 82, 1668 (1999).

[6] C.G. Durfee, et. al., Phys. Rev. Lett., 83, 2187 (1999). 
[7] Y. Tamaki, et. al., Phys. Rev. A, 62, 063802 (2000).

[8] E. Takahashi, et. al., Phys. Rev. A, 66, 021802( R) (2002).

[9] D.E. Moncton and W.S. Graves, Proc. 2003 Synchr. Rad. Instr. Conf., SF (2003).

[10] G. Lambert, et. al., Proc. 2004 FEL Conf., Trieste (2004). 